\title{
AN INVESTIGATION OF THE RELATIONSHIP BETWEEN THE eNOS GENE POLYMORPHISM AND DIAGNOSED MIGRAINE
}

\author{
Güler $\mathrm{S}^{1, *}$, Gürkan $\mathrm{H}^{2}$, Tozkir $\mathrm{H}^{2}$, Turan $\mathrm{N}^{3}$, Çelik $\mathrm{Y}^{1}$
}

\begin{abstract}
*Corresponding Author: Sibel Güler, M.D., Department of Neurology, Trakya University Faculty of Medicine, Balkan Yerleşkesi, 22030 Edirne, Turkey. Tel: +90-284-236-49-81. Fax: +90-284-223-42-03. E-mail: drsibleguler@, yahoo.com
\end{abstract}

\begin{abstract}
We investigated the phenotype-genotype association of the following endothelial nitric oxide synthase (eNOS) gene polymorphisms, rs 743506, rs2070744, rs 1799983, rs 180079, rs3918226, rs207468799 and rs 148554851, in patients suffering from migraine living in Edirne, Turkey. A total of 175 individuals, who had been diagnosed with migraine between April 2013 and December 2013, at the Neurology Department, Trakya University Medical Faculty, Edirne, Turkey, and 125 healthy controls were recruited. The above gene polymorphisms were analyzed from genomic DNA in both patient and control groups, using the pyro-sequencing method.
\end{abstract}

The eNOS rs1799983 TT genotype frequency in migraine patients who had a headache duration of longer than 24 hours was statistically significantly higher than in patients who had migraine attacks that lasted under 24 hours $(p=0.047)$. In terms of the AGGTGGA haplotype, the severity of headache was statistically significant, and was found to be severe in $61.0 \%$ ( $p=0.0001)$. Also in terms of the AGGTGGA haplotype, the duration of headache was statistically significant, and was $>24$ hours in $56.0 \%$ of patients $(p=0.008)$.

\footnotetext{
Department of Neurology, Trakya University Faculty of Medicine, Edirne, Turkey

2 Department of Genetics, Trakya University Faculty of Medicine, Edirne, Turkey

${ }^{3}$ Department of Biostatistics, Trakya University Faculty of Medicine, Edirne, Turkey
}

In our study, there was no significant genotypephenotype relationship between eNOS rs 743506 , rs2070744, rs1799983, rs180079, rs3918226, rs207468799 and rs148554851 gene polymorphisms and migraine patients with and without aura living in Edirne, Turkey. The AGGTGGA haplotype constitutes a risk in terms of the severity and the duration of headaches in patients with migraine. This risk is significantly higher in patients with migraine with aura than patients with migraine without aura.

Keywords: Migraine; Aura; Clinical features; Endothelial nitric oxide synthase (eNOS) gene; Polymorphisms.

\section{INTRODUCTION}

Migraine is a neurological disease that affects approximately $12.0-16.0 \%$ of the population. It leads to specific disabling conditions and has a genetic component [1]. Despite its high prevalence, the complex pathogenetic mechanisms of migraine remain unclear. However, the significance of nitric oxide (NO) in migraine pathogenesis has been reported in some studies. In essence, NO plays an important role in cerebral blood flow regulation and is involved in the activation of nociceptors in the trigeminovascular system and the release of vasoactive neuropep-tides during the neurogenic inflammatory response $[2,3]$ causing vascular homeostasis, blocking of platelet adhesion and aggregation, inhibition of migration and proliferation of leukocyte and vascular muscle cells [4]. Thus, NO makes a 
significant contribution to antiatherogenic features in the endothelium.

According to recent studies, genes encoding endothelial function regulators are indicated as significant candidate genes in individuals susceptible to migraine. Therefore, it has been claimed that gene polymorphisms for endothelial nitric oxide synthase gene (eNOS), are possible genetic factors involved in migraine [2]. It has been indicated that there is a strong association between NO and the pathophysiology of migraine and aura [5]. During the headache phase, an increase of platelets and administration of $\mathrm{NO}$ as an exogen makes the headache worse [5]. A Glu 268Asp amino acid change and point mutation (G894T, rs1799983) leads to a guanine to thymine change on exon 7 and nucleotide (nt) 894 in the eNOS gene, and this is related to reduced basal NO production. Another polymorphism (-786T $>C$, rs1800779) leads to a thymine to cytosine change on the eNOS 5 , promoter region $-786 \mathrm{nt}$, which causes a reduction of both eNOS gene promoter activity and basal NO production [2]. To date, the studies investigating the association between migraine and eNOS gene polymorphisms have focused on eNOS rs743506, rs2070744, rs1799983, rs 180079, rs3918226, rs207468799 and rs148554851 [5].

We believe that it is important to determine the genetic basis of the common primary headache that leads to migraine, since the latter creates disabling circumstances for people with regard to their social and working life. Therefore, we investigated phenotype-genotype association between eNOS rs 743506 , rs2070744, rs1799983, rs180079, rs3918226, rs207468799 and $r s 148554851$ gene polymorphisms, which we suggest are candidate markers of susceptibility to migraine with and without aura.

\section{MATERIALS AND METHODS}

Participants. A total of 300 individuals, comprising 175 migraine patients [ $25(14.3 \%)$ males, 150 (85.7\%) females] and 125 controls [ 28 (22.4\%) males and $97(77.6 \%)$ females] were recruited. Local ethics committee approval was obtained from the Trakya University Medical Faculty Ethics Committee on 13 February 2013, and the official writing number is $2013 / 26$. The participants with migraine had all been referred to the Trakya University Medical Faculty Neurology Policlinic, Edirne, Turkey between
January 2013 and December 2013, and had been diagnosed according to the International Classification of Headache Disorders (ICHD-II) criteria [6]. The need to recruit 103 cases to both patient and control groups was calculated on the basis that polymorphisms might be observed as 0.34 in the control group, and an odds ratio (OR) of 2.21, so in order to find an eNOS polymorphism association at $80.0 \%$ power, 300 people consisting of 175 patients and 125 controls were recruited. Patients who had cardiovascular, renal, hepatic, gastrointestinal, pulmonary, endocrine, oncologic, autoimmune, respiratory and inflammatory diseases were excluded. Controls were selected randomly among people who had no cardiovascular, renal, hepatic, gastrointestinal, pulmonary, endocrine, oncologic, autoimmune, respiratory, inflammatory and psychiatric diseases.

Genotype determination. Peripheral blood samples from both patients and controls were drawn into $2 \mathrm{~mL}$ EDTA tubes. DNA isolation from peripheral blood samples were performed using Qiagen DNA isolation kits (EZ1 ${ }^{\circledR}$ DNA Blood $200 \mu \mathrm{L} \mathrm{Kit;}$ Qiagen, Hilden, North Rhine-Westphalia, Germany) with an EZ1 Advanced XL (Qiagen) Nucleic Acid Isolation system. Consequently, DNA concentration and purity of isolated DNA samples was measured using a NanoDrop device [NanoDrop 2000C; Thermo Fisher Scientific Inc., Wilmington, MA, USA]. After measurement of concentration and purity, amplification polymerase chain reaction (PCR) for pyro-sequencing was performed according to the manufacturer's recommended PCR protocol, using a PyroMark PCR kit (Qiagen) and primers in a PyroMark Custom Assay Kit (Qiagen) for detection of each polymorphism, such as rs743506, rs207468799, rs3918226, rs2070744, rs1799983, rs148554851 and rs180079. Amplification PCR performed with initial denaturation at $95{ }^{\circ} \mathrm{C}$ for $15 \mathrm{~min}$., followed by 45 cycles at $94{ }^{\circ} \mathrm{C}$ for 30 seconds, $60^{\circ} \mathrm{C}$ for 30 seconds, $72^{\circ} \mathrm{C}$ for 30 seconds, and a final extension $72{ }^{\circ} \mathrm{C}$ for $10 \mathrm{~min}$.

After PCR amplification, PCR products were pyro-sequenced to detect each polymorphism using sequencing primers from a PyroMark Custom Assay Kit, according to the manufacturer's instructions (PyroMark Q24 System; Qiagen). The results were then analyzed using the PyroMark Q24 software system (Qiagen), and the genotypes for polymorphisms were determined using samples from both controls and patients (Qiagen). 
Statistical analyses. Statistical evaluation was performed using the Statistical Package for the Social Sciences (SPSS Inc., Chicago, IL, USA) (SPSS v21) statistics software. One sample KolmogorovSmirnov test was used to assess the eligibility for normal distribution of measured data, and since the data did not show a normal distribution, the Mann Whitney U test and the Kruskal-Wallis analysis of variance were used for comparison between groups. Pearson's $\mathrm{c}^{2}$ test, Fisher's exact $\mathrm{c}^{2}$ analysis and the Kolmogorov-Smirnov two-sample test were used to analyze qualitative data. Median (minimum-maximum) values and mean value \pm standard deviation (SD) were determined as descriptive statistics. The significance limit was set at $p<0.05$ for all statistics.

\section{RESULTS}

Migraine with aura was detected in 58 patients and migraine without aura was detected in 117 patients; the difference was statistically significantly $(p<0.001)$. There was no significant difference in terms of average age of both patient and control groups, or in terms of body mass index (BMI) $(p>0.05)$. Moreover, there was no statistically significant association between time after migraine diagnosis, frequency, family history of diagnosis of migraine, stroke and coronary artery disease, and allele-genotype frequency of eNOS $r s 743506$, rs207468799, rs2070744, rs1799983, rs148554851, $r s 180079$ and $r s 3918226$ gene polymorphisms ( $p$ $>0.05$ ). Migraine attack duration was longer than 24 hours in $77.2 \%$ of migraine patients with aura, and in $45.3 \%$ of patients with migraine without aura ( $p=$ $0.001)$. There was a statistically significant association between the eNOS rs1799983 TT genotype frequency and headache duration of longer than 24 hours $(70.0 \%)$ and headache duration of shorter than 24 hours (30.0\%) $(p=0.047)$. The GG genotype frequency of the eNOS rs 743506 gene polymorphism was detected to be 70.0 , 30.0 and $0.0 \%$ in migraine patients with severe, mild and light headaches, respectively $(p=0.381)$. The GG genotype frequency of the eNOS rs743506 was determined to be $70.0 \%$ in patients with headache duration of longer than 24 hours, and as $30.0 \%$ in patients with headache duration of shorter than 24 hours. Although there was no statistically significant difference, the GG

Table 1. Clinical characteristics of the study participants.

\begin{tabular}{|c|c|c|c|c|}
\hline Parameters & Controls & Migraine With Aura (\%) & Migraine Without Aura (\%) & $p$ Value \\
\hline Number & 125 & 117 & 58 & $<0.001$ \\
\hline Age (years) & $37.22 \pm 14.30$ & $37.71 \pm 11.80$ & $39.97 \pm 13.40$ & NS \\
\hline $\mathrm{BMI}\left(\mathrm{kg} / \mathrm{m}^{2}\right)$ & $25.68 \pm 4.72$ & $25.59 \pm 4.91$ & $27.30 \pm 5.01$ & NS \\
\hline Family history (\%) & - & $56.4 \%$ & $56.1 \%$ & NS \\
\hline \multicolumn{5}{|l|}{ Frequency $(n, \%)$} \\
\hline $1-3 /$ month & - & $62(53.0)$ & $24(42.1)$ & NS \\
\hline $3-5 /$ month & - & $36(30.8)$ & $19(33.3)$ & NS \\
\hline 5-10/month & - & $16(17.3)$ & $11(19.3)$ & NS \\
\hline $10-15 /$ month & - & $3(2.6)$ & $3(5.3)$ & NS \\
\hline \multicolumn{5}{|l|}{ Intensity (\%) } \\
\hline mild & - & $2(1.7)$ & $0(0.0)$ & NS \\
\hline moderate & - & $45(38.5)$ & $17(29.8)$ & NS \\
\hline severe & - & $70(59.8)$ & $40(70.2)$ & NS \\
\hline \multicolumn{5}{|l|}{ Duration (\%) } \\
\hline$<12$ hours & - & $28(23.9)$ & $8(14.0)$ & NS \\
\hline 12-24 hours & - & $36(30.8)$ & $5(8.8)$ & NS \\
\hline$>24$ hours & - & $53(45.3)$ & $44(77.2)$ & $<0.001$ \\
\hline
\end{tabular}

BMI: body mass index; NS: no statistics were computed. Values are presented as mean \pm SD (standard deviation) or $\%$ number of subjects. 
Table 2. Genotype and allele frequencies of eNOS rs743506, rs207468799, rs2070744, rs1799983, rs148554851, rs 180079, rs3918226, rs207468799 and rs148554851 gene polymorphisms in the control and patient groups.

\begin{tabular}{|c|c|c|c|c|c|c|c|c|c|}
\hline eNOS SNPs & \multicolumn{3}{|c|}{ Genotype $n(\%)$} & Total $n(\%)$ & \multicolumn{4}{|c|}{ Allele $n(\%)$} & \multirow[t]{2}{*}{ Total $n(\%)$} \\
\hline rs743506 & AA & $\mathrm{AG}$ & GG & & A & G & $\mathrm{C}$ & $\mathrm{T}$ & \\
\hline Patients & $104(59.4)$ & $61(34.9)$ & $10(5.7)$ & $175(100.0)$ & $269(76.9)$ & $81(23.1)$ & - & - & $350(100.0)$ \\
\hline Controls & $64(51.2)$ & $49(39.2)$ & $12(9.6)$ & $125(100.0)$ & $177(70.8)$ & $73(29.2)$ & - & - & $250(100.0)$ \\
\hline$p$ Value & \multicolumn{4}{|c|}{$0.252^{\mathrm{a}}$} & \multicolumn{5}{|c|}{$0.629^{\mathrm{a}}$} \\
\hline rs207468799 & GG & GA & $\mathrm{AA}$ & & A & G & $\mathrm{C}$ & $\mathrm{T}$ & \\
\hline Patients & $175(100.0)$ & $0(0.0)$ & $0(0.0)$ & $175(100.0)$ & - & $350(100.0)$ & - & - & $350(100.0)$ \\
\hline Controls & $125(100.0)$ & $0(0.0)$ & $0(0.0)$ & $125(100.0)$ & - & $250(100.0)$ & - & - & $250(100.0)$ \\
\hline$p$ Value & \multicolumn{4}{|c|}{ no statistics were computed } & \multicolumn{5}{|c|}{ no statistics were computed } \\
\hline rs3918226 & GG & GA & $\mathrm{AA}$ & & A & G & $\mathrm{C}$ & $\mathrm{T}$ & \\
\hline Patients & $151(86.3)$ & $23(13.1)$ & $1(0.69)$ & $175(100.0)$ & $25(7.1)$ & $325(92.9)$ & - & - & $350(100.0)$ \\
\hline Controls & $108(86.4)$ & 17 (13.6) & $0(0.0)$ & $125(100.0)$ & $17(6.8)$ & $233(93.2)$ & - & - & $250(100.0)$ \\
\hline$p$ Value & \multicolumn{4}{|c|}{$1.000^{b}$} & \multicolumn{5}{|c|}{$1.000^{\mathrm{b}}$} \\
\hline rs2070744 & $\mathrm{CC}$ & $\mathrm{CT}$ & TT & & A & G & $\mathrm{C}$ & $\mathrm{T}$ & \\
\hline Patients & $18(10.3)$ & $86(49.1)$ & $71(40.6)$ & $175(100.0)$ & - & - & $122(34.9)$ & $228(65.1)$ & $350(100.0)$ \\
\hline Controls & $12(9.6)$ & $67(53.6)$ & $46(36.8)$ & $125(100.0)$ & - & - & $91(36.4)$ & 159 (63.6) & $250(100.0)$ \\
\hline$p$ Value & \multicolumn{4}{|c|}{$0.747^{a}$} & \multicolumn{5}{|c|}{$0.883^{a}$} \\
\hline rs1799983 & GG & GT & TT & & A & G & $\mathrm{C}$ & $\mathrm{T}$ & \\
\hline Patients & 99 (56.6) & $66(37.7)$ & $10(5.7)$ & $175(100.0)$ & - & $264(75.4)$ & - & $86(24.6)$ & $350(100.0)$ \\
\hline Controls & $59(47.2)$ & $58(46.4)$ & $8(6.4)$ & $125(100.0)$ & - & $176(70.4)$ & - & 74 (29.6) & $250(100.0)$ \\
\hline$p$ Value & \multicolumn{4}{|c|}{$0.272^{\mathrm{a}}$} & \multicolumn{5}{|c|}{$0.428^{a}$} \\
\hline rs 148554851 & GG & GA & AA & & A & G & $\mathrm{C}$ & $\mathrm{T}$ & \\
\hline Patients & $175(100.0)$ & $0(0.0)$ & $0(0.0)$ & $175(100.0)$ & - & $350(100.0)$ & - & - & $350(100.0)$ \\
\hline Controls & $125(100.0)$ & $0(0.0)$ & $0(0.0)$ & $125(100.0)$ & - & $250(100.0)$ & - & - & $250(100.0)$ \\
\hline$p$ Value & \multicolumn{4}{|c|}{ no statistics were computed } & \multicolumn{5}{|c|}{ no statistics were computed } \\
\hline rs1800779 & GG & GA & $\mathrm{AA}$ & & A & G & $\mathrm{C}$ & $\mathrm{T}$ & \\
\hline Patients & $16(9.1)$ & $89(50.9)$ & $70(40.0)$ & $175(100.0)$ & $229(65.4)$ & $121(34.6))$ & - & - & $350(100.0)$ \\
\hline Controls & $12(9.6)$ & $67(53.6)$ & $46(36.8)$ & $125(100.0)$ & $159(63.6)$ & $91(36.4)$ & - & - & $250(100.0)$ \\
\hline$p$ Value & \multicolumn{4}{|c|}{$0.854^{\mathrm{a}}$} & \multicolumn{5}{|c|}{$0.883^{\mathrm{a}}$} \\
\hline
\end{tabular}

eNOS SNPs: endothelial nitric oxide synthase single nt polymorphisms.

a Pearson's c ${ }^{2}$ test.

${ }^{\mathrm{b}}$ Kolmogorov-Smirnov two-sample test.

genotype frequency was distinctly higher in patients with shorter duration and lighter headache attacks ( $p$ $=0.359$ ). The TT genotype frequency of the eNOS $r s$ 2070744 gene polymorphism was determined to be 69.0, 20.0 and $2.0 \%$ in migraine patients with severe, mild and light headaches, respectively, but there was no statistically significant difference $(p=0.171)$. The
AA genotype frequency of the eNOS $r s 180079$ gene polymorphism was also detected as $68.6,28.6$ and $2.9 \%$ in migraine patients with severe, mild and light headaches, respectively, but there was no statistically significant association ( $p=0.205)$. Other clinical features of individuals in the patient and control groups are summarized in Table 1. 
Table 3. Genotype frequencies of eNOS $r s 743506, r s 207468799$, rs2070744 and rs1799983, rs148554851, rs180079, rs3918226, rs207468799 and rs 148554851 ene polymorphisms in migraine with and without aura patients and control group.

\begin{tabular}{|c|c|c|c|c|c|}
\hline eNOS SNPs & Genotype & Without Aura $n(\%)$ & With Aura $n(\%)$ & Controls $n(\%)$ & $p$ Value \\
\hline rs743506 & $\begin{array}{l}\text { AA } \\
\text { AG } \\
\text { GG }\end{array}$ & $\begin{array}{c}67(39.9) \\
43(39.1) \\
3(13.6)\end{array}$ & $\begin{array}{c}37(22.0) \\
18(16.4) \\
7(31.8)\end{array}$ & $\begin{array}{l}64(38.1) \\
49(44.5) \\
12(54.5)\end{array}$ & $0.225^{\mathrm{a}}$ \\
\hline rs207468799 & $\begin{array}{l}\text { GG } \\
\text { GA } \\
\text { AA }\end{array}$ & $\begin{array}{c}117(39.0) \\
0(0.0) \\
0(0.0)\end{array}$ & $\begin{array}{c}58(19.3) \\
0(0.0) \\
0(0.0)\end{array}$ & $\begin{array}{c}125(41.7) \\
0(0.0) \\
0(0.0)\end{array}$ & NS \\
\hline rs2070744 & $\begin{array}{l}\mathrm{CC} \\
\mathrm{CT} \\
\mathrm{TT}\end{array}$ & $\begin{array}{l}11(36.7) \\
60(39.2) \\
46(39.3)\end{array}$ & $\begin{array}{c}7(23.3) \\
26(17.0) \\
25(21.4)\end{array}$ & $\begin{array}{c}12(40.0) \\
67(43.89) \\
46(39.3)\end{array}$ & $0.858^{\mathrm{a}}$ \\
\hline rs1799983 & $\begin{array}{l}\text { GG } \\
\text { GT } \\
\text { TT }\end{array}$ & $\begin{array}{c}66(39.2) \\
46(37.1) \\
5(27.8)\end{array}$ & $\begin{array}{c}33(20.9) \\
20(16.1) \\
5(27.8)\end{array}$ & $\begin{array}{c}59(37.3) \\
58(46.8) \\
8(44.8)\end{array}$ & $0.398^{\mathrm{a}}$ \\
\hline rs 148554851 & $\begin{array}{l}\text { GG } \\
\text { GA } \\
\text { AA }\end{array}$ & $\begin{array}{c}117(39.0) \\
0(0.0) \\
0(0.0)\end{array}$ & $\begin{array}{c}58(19.3) \\
0(0.0) \\
0(0.0)\end{array}$ & $\begin{array}{c}125(41.7) \\
0(0.0) \\
0(0.0) \\
\end{array}$ & NS \\
\hline rs 180079 & $\begin{array}{l}\text { GG } \\
\text { GA } \\
\text { AA }\end{array}$ & $\begin{array}{l}10(35.7) \\
61(39.1) \\
46(39.7)\end{array}$ & $\begin{array}{r}6(21.4) \\
28(17.9) \\
24(20.7)\end{array}$ & $\begin{array}{l}12(42.9) \\
67(42.9) \\
46(39.7)\end{array}$ & $0.963^{\mathrm{a}}$ \\
\hline rs3918226 & $\begin{array}{l}\text { GG } \\
\text { GA } \\
\text { AA }\end{array}$ & $\begin{array}{c}99(38.2) \\
18(45.0) \\
0(0.0)\end{array}$ & $\begin{array}{c}52(20.1) \\
5(12.5) \\
1(100.0)\end{array}$ & $\begin{array}{c}108(41.7 \\
17(42.5) \\
0(0.0)\end{array}$ & $1.000^{\mathrm{b}}$ \\
\hline
\end{tabular}

eNOS SNPs: endothelial nitric oxide synthase single nt polymorphisms; NS: no statistics were computed.

a Pearson's c ${ }^{2}$ test.

${ }^{\mathrm{b}}$ Kolmogorov-Smirnov two-sample test.

There were no statistically significant differences in association of genotype and allele frequencies of eNOS rs 743506, rs207468799, rs2070744, rs1799983, rs148554851, rs180079, rs3918226, rs207468799 and $r s 148554851$ gene polymorphisms between the patient and control group $(p>0.05)$, as shown in Table 2 . No statistically significant differences in genotype frequencies of the above gene polymorphisms were detected between the migraine with and without aura patient group and the control group $(p>0.05)$, as shown in Table 3 .

Genotypes of patients were evaluated and haplotype analysis was performed. Unlike other studies, a total of seven polymorphisms were studied. This increases the frequency of heterozygous genotypes. Haplotype analysis was performed in individuals with a homozygous genotype of seven polymorphism (wild type/mutant type). Haplotype analysis could

Table 4. Haplotype analysis results.

\begin{tabular}{|c|c|c|c|c|c|}
\hline & Haplotypes & Without Aura $\boldsymbol{n}(\mathbf{\%})$ & With Aura $\boldsymbol{n}(\mathbf{\%})$ & Controls $\boldsymbol{n}(\mathbf{\%})$ & $\boldsymbol{p}$ Value \\
\hline H1 & AGGTGGA & $25(61.0)$ & $16(39.0)$ & $31(43.1)$ & $1.000^{\mathrm{a}}$ \\
\hline H2 & AGGCGGG & $1(3.6)$ & $1(5.3)$ & $2(5.7)$ & ND \\
\hline H3 & GGGTGGA & $1(3.6)$ & $0(0.0)$ & $1(2.9)$ & ND \\
\hline H4 & GGGCTGG & $1(3.6)$ & $0(0.0)$ & $1(2.9)$ & $1.000^{\mathrm{a}}$ \\
\hline H5 & GGACTGG & $0(0.0)$ & $1(5.3)$ & $0(0.0)$ & $1.000^{\mathrm{a}}$ \\
\hline H6 & AGGTTGA & $0(0.0)$ & $1(5.3)$ & $0.0)$ & $1.000^{\mathrm{a}}$ \\
\hline
\end{tabular}

${ }^{a}$ Kolmogorov-Smirnov two-sample test. 
not be performed in individuals with a heterozygous genotype because the genotypes of the parents of patient and control groups were not known. According to our study results, six haplotypes in the patient group and four haplotypes in the control group were created (Table 4).

The AGGTGGA haplotype was detected in 41 $(56.9 \%)$ migraine patients, while only in $31(43.1 \%)$ people in the control group. No significant difference was found between patient and control groups in terms of frequency of carriers of this haplotype ( $p$ $>0.05$ ). The AGGCGGG hap-lotype was detected in two people each in the patient and control groups. The GGGTGGA and GGGCTGG haplotypes were observed in one person each in the patient and control groups. The GGACTGG and AGGTTGA haplotypes were detected only in one person in the patient group but not in the control group $(p>0.05)$. The AGGTGGA haplotype was observed in $16(39.0 \%)$ patients with migraine with aura and $25(61.0 \%)$ patients with migraine without aura; the difference was not statistically significant $(p>0.05)$. Two $(4.9 \%)$ patients with migraine with the AGGTGGA haplotype had mild headaches, whereas 14 (34.1\%) had moderate and $25(61.0 \%)$ had severe headaches. In terms of the AGGTGGA haplotype, the severity of headache was statistically significant, and was found to be severe in $61.0 \%$ ( $p=0.0001)$. Nine $(22.0 \%)$ of the patients with migraine with the AGGTGGA haplotype had episode duration of $<12$ hours, while nine $(22.0 \%)$ patients had episode duration between 12 and 24 hours, and 23 patients had episode duration of $>24$ hours. There was a statistically significant difference in headache duration in terms of AGGTGGA haplotype.

There was no statistical difference between the AGGTGGA haplotype patients with migraine and with or without aura, in terms of monthly headache frequency $(p>0.05)$. None $(0.0 \%)$ of the patients with migraine aura and the AGGTGGA haplotype had mild headaches, while two had moderate and $14(87.5 \%)$ had severe headaches. Two (8.0\%) of the patients without migraine had mild headaches, while $12(48 \%)$ had moderate and $11(44.0 \%)$ had severe headaches. Between the AGGTGGA haplotype of migraine with or without aura, there was a significant difference in terms of severity of headache, $87.5 \%$ of patients with migraine with aura had severe headaches $(p=0.050)$. Three $(18.8 \%)$ of the patients with migraine with aura with AGGTGGA haplotype had episode duration of $<12$ hours, none of them had episode duration between 12 and 24 hours, and $13(81.3 \%)$ had episode duration of $>24$ hours. Six $(24.0 \%)$ of the patients with migraine without aura had episode duration of $<12$ hours, nine $(22.0 \%)$ had episode duration between 12 and 24 hours, and 23 $(56.1 \%)$ had episode duration of $>24$ hours. Although there was no statistically significant difference in AGGTGGA haplotype between patients with migraine with and without aura, the duration of headaches was $>24$ hours in $81.0 \%$ of the patients with clinical aura $(p=0.072)$.

\section{DISCUSSION}

Migraine is a neurovascular disorder that particularly affects females and may cause significant disability [7]. The pathogenesis of migraine is fairly complex, and is not yet clear. Several previous studies have investigated candidate genes affecting endothelial function, such as eNOS, inducible NO and vascular endothelial growth factor (VEGF), and studies investigating gene-gene interaction are more effective in increasing our understanding of the genetic basis of migraine [8]. Genetic patterns can change in terms of ethnic and racial properties. Thus, the results of gene polymorphism studies conducted in different countries and in people with different ethnicity and race can differ from one another. Our study examined patients and healthy controls living in the city of Edirne in the Thrace region of Turkey. There are few immigrants in this region, and no massive population exchange has occurred, meaning that demographic properties have been preserved. In the present study, we proposed an association between eNOS polymorphisms and frequency, severity and duration of headaches.

In previous studies, it has been shown that healthy individuals suffering from migraine-like headaches, when they use medications containing NO [9]. Moreover, administration of NO inhibitors due to their anti-migraine effects, is consistent with the concept of abnormal NO formation contributing to the pathogenesis of migraine [10]. In this respect, while NO formation is mostly attributed to the activity of three different NOS [endothelial (eNOS), neuronal (nNOS) and inducible NOS (iNOS)], it is possible that the excessive amounts of $\mathrm{NO}$, possibly contributing to migraine, are derived from increased 
expression and activity of iNOS $[11,12]$. Although iNOS is primarily regulated at the transcriptional level, the association of the promoter region of the iNOS gene with migraine susceptibility was also examined $[13,14]$.

Haplotype analysis is important, because the analysis of genetic combinations in the involved region and effects of genetic markers can provide further information, if they are examined properly [13]. Mansur et al. [12] studied the functional and clinical association of iNOS genetic polymorphism [the $\mathrm{C}^{-}$ ${ }^{1026} \mathrm{~A}$ polymorphism (rs2779249) in the promoter region, and the G2087A polymorphism (rs2297518) in exon 16] with migraine with or without aura, and also the association of iNOS haplotypes with migraine [12]. While the H4 haplotype is associated with migraine with aura (that combines both A alleles), the presence of the A allele and $\mathrm{H} 4$ haplotype for the G2087A polymorphism has been shown to increase the tendency. Considered together, these findings reveal that the A allele for the G2087A polymorphism is a predisposing factor for aura development in patients with migraine. It has been shown that the tendency to develop migraine with aura increases when the $\mathrm{A}$ allele is combined with the $\mathrm{C}^{-1026} \mathrm{~A}$ polymorphism [12]. Thus, the association between iNOS activity and development of migraine was detected. It has also been reported that patients, who are at-risk of developing migraine with aura, may be detected earlier and can benefit from selective or non selective iNOS inhibitors [12]. Therefore, the individuals who have genetic expression and increased iNOS activity associated with genetic markers, are expected to benefit more from treatment with selective iNOS inhibitors.

In some migraine cases, in addition to nausea, vomiting, photophobia and phonophobia, temporary neurological symptoms that occur before the onset of headaches, and known as aura, have been observed [15]. Migraine with aura is especially associated with cerebrovascular and cardiovascular disease risk. There is much evidence to suggest that NO is active in the pathophysiology of migraine and aura, and it exerts its effects via control of cerebral blood flow, nociceptor activation in the trigemino-vascular system and vasoactive neuropeptide release during neurogenic inflammation response. It has been reported that genes responsible for regulation of endothelial function are important in people who are susceptible to migraine. Therefore, it has been claimed that eNOS gene polymorphisms create a genetic predisposition to migraine.

Migraine and cerebrovascular disease, especially association with ischemic stroke, is one of the most confusing neurological problems. Although the two disorders have differing epidemiologies, case-control and population-based studies have shown that there is a significant association between ischemic stroke and migraine. Indeed, migraine with aura in particular, is an independent risk factor for ischemic stroke [16]. Previous studies have shown that gliceryl trinitrate can cause migraine-like headaches by turning into NO, and L-MMA (L-nitromonomethylarginine), a potent anti-migraine and NOS inhibitor, is effective in those cases [17]. However, the mechanism by which NO triggers migraine has not been identified. In particular, an increased risk of ischemic stroke and cardiovascular diseases should be the focus in female patients with migraine-related endothelial dysfunction and migraine with aura [18]. In our study, we detected no statistically significant association between eNOS rs743506, rs207468799, rs2070744, rs1799983, rs148554851, rs 180079 and rs3918226 gene polymorphisms and ischemic stroke, cardiovascular and cerebral medical history.

The correlation between vasodilation, headache and, thereby, NO presence, has been shown in previous studies. Thus, the important role of detected genetic variations in eNOS in the pathogenesis of migraine with aura has been reported [19]. In contrast, we did not detect a similar correlation for migraine with aura. It has been indicated that differences in ethnic groups plays a main role in distribution of eNOS variants [20]. Knowledge of the differences in haplotype frequency and association between variants is thought to provide a better understanding of the role of eNOS in cardiovascular and cerebral diseases.

We determined significant differences in the distributions of eNOS haplotypes in patients with migraine, with and without aura. The GA genotype of the tag single nt polymorphism (tagSNP) rs 743506 showed protective effects and the haplotypes " $\mathrm{C} \mathrm{C}$ a Glu G" and " $\mathrm{C} \mathrm{C}$ b Glu G" were associated with aura in migraine patients. This shows that eNOS genetic variations can increase aura progression. However, the decision-making mechanism is not certain. Furthermore, it has been claimed that different isoforms, known as nNOS, and their polymorphisms, may be capable of playing a role in migraine progression [5]. 
Haplotype analysis, combinations of genetic markers within a chromosome cluster location, is valuable because it provides a more powerful approach to genetic studies. Because only the analysis of a single nt can be performed each time, it is possible to eliminate inconsistencies [21]. While the association between individual eNOS polymorphisms and concentration changes of nitrate in blood cannot be detected, Metzger et al. [22] have shown that the specific and unifying eNOS gene alleles of the selected eNOS haplotype, is associated with low nitrate concentrations in blood of apparently healthy individuals. The haplotype-specific expression pattern has also been shown for eNOS, that was determined by variants of the eNOS gene [23]. Intensive effort has been made to verify the clinical markers of NO formation $[24,25]$. Nitric oxide-induced soluble guanylyl cyclase is one of the most important biological effects produced by NO, thereby, the concentration of cyclic guanosine 3,5 monophosphate (cGMP) increases. Previous studies reported that an eNOS poly-morphism alone has no important effect on NO formation, however, combined with specific haplotypes, it has been reported to have important effect. Thus, it significantly does not affect in vivo bioavailability of NO. In our study, we demonstrated that the AGGTGGA haplotype constitutes a risk in patients with migraine in terms of severity and duration, and also that risk is higher in patients with migraine with aura. Consistent with the literature, specific haplotypes have higher and more specific effect on NO formation, and this constitutes one of the most important results of our study.

Plasma concentrations of cGMP have been reported to have no significant effect on eNOS polymorphisms and its haplotypes [26]. Considering the specific eNOS genotypes alone, it is uncertain whether or not eNOS haplotypes have an important contribution to the formation of NO. In the literature, it has been reported to have an important relationship in terms of NO formation between C-4b-Glu hap-lotype of eNOS and low nitrite/nitrate concentrations in blood [22]. In another study, the C-4b-Glu haplotype has been shown to be common in Black individuals with lower nitrite concentrations in plasma and whole blood compared to individuals with higher nitrite concentrations in plasma. These findings were similar to the results of studies in Caucasian individuals. Rather than ethnic classification, this condition shows that the genetic markers have a more important role in NO formation [27]. Another study that shows no association in healthy individuals between C-4bGlu haplotype and development of cardiovascular disease, suggests studying C-4b-Glu haplotypes in individuals with cardiovascular disease.

In the literature, the effects of eNOS tagSNPs rs3918188, rs743506 and rs7830 haplotypes to NO bio-availability in Black individuals have been studied [28]. The frequency of the CA genotype for tagSNP rs7830 haplotype and C-G-A haplotype has been found to be higher in individuals with lower blood nitrate concentration compared to individuals with higher blood nitrate concentration, although there was no significant difference in genotype results according to several investigations after multivariate analyses [28]. The effects of rs 7830 tagSNP on blood nitrate concentrations have been shown and it was considered that a tagSNP may be a marker to indicate risk factors for cardiovascular disease.

Our study is the first to have investigated an association between seven eNOS gene polymorphisms and migraine. In previous studies of eNOS polymorphisms in the Edirne region, no associations have been detected between migraine and eNOS variable number of tandem repeats (VNTR) (eNOS 4a/b) [29], and between stroke patients and a Glu298Asp eNOS polymorphism [30]. In our study, the lack of a significant association between genotype and allele frequency of the eNOS rs 743506, rs207468799, rs2070744, rs 1799983, rs148554851, rs180079 and rs3918226 gene polymorphisms in migraine patients and the control group may arise from ethnic differences in the populations.

Although migraine pathogenesis is not exactly understood, genetic and environmental factors are known to have an effect. We hypothesize that our results provide significant data regarding the Edirne geographical region. When our study is evaluated alongside other studies that have been carried out with different populations and ethnic groups, it allows us to better understand the role of eNOS polymorphisms in migraine pathogenesis. Determination of genetic factors in migraine etiopathogenesis can inform important changes in treatment strategies. Therefore, it has been claimed that the loss of productivity in the workplace and the social disability resulting from migraine, which negatively affects quality of life, can be considerably reduced. 
In conclusion, this study demonstrated that there was no significant association between the eNOS rs743506, rs207468799, rs2070744, rs1799983, rs 148554851, rs 180079 and $r s 3918226$ gene polymorphisms and migraine with or without aura, whether complicated or not, in the Turkish individuals we examined. Our results showed that the eNOS rs 1799983, rs 743506, rs2070744 and rs 180079 gene polymorphisms can be a risk factor in migraine duration and severity in particular. Moreover, in our study, according to haplotype analysis results, the AGGTGGA haplotype has been shown to increase the severity and the duration of migraine more significantly in patients with migraine with aura, compared to patients with migraine without aura. The AGGTGGA haplotype increases migraine-related disability and deterioration in quality of life, and it constitutes one of the most important results in our study. This condition may be associated with low blood nitrate concentrations. As with cardiovascular disease, abnormalities of NO formation may be a risk factor for the development of debilitating migraine with aura. But, these data should be built on, via studies in different populations and that use a higher number of patients.

The relationship between migraine and the NOTCH4 gene, mapped on chromosome 6p21.3, has been intensively investigated [31,32]. While no significant association between R1346P and G835V NOTCH4 gene poly-morphisms and patients with migraine with or without aura and control groups has been detected, a significant association between migraine attack duration and severity has been reported [33]. Similarly, we did not detect any significant association between seven polymorphisms in the eNOS gene and migraine with or without aura. However, when the results are evaluated alongside clinical findings, we hypothesize that the TT genotype in the eNOS rs 1799983 gene polymorphism could be a risk factor in migraine with aura patients who have headache duration of longer than 24 hours.

Two studies, conducted in Austria and Spain, reported that there was no significant association between eNOS rs1800779 and rs1799983 gene polymorphisms and migraine [2,3] However, our data suggest that the eNOS rs1799983 gene polymorphism could have an important role in determining migraine attack duration and the intensity of these attacks. The most important aim of migraine treatment should be the reduction of duration and severity of the attacks. It has been claimed that treatment plan and medicine selection should be carried out on that basis. Our results support the hypothesis that vascular genetic factors play an important role in migraine pathogenesis. As a result, the eNOS rs743506, rs2070744, rs 180079, and especially rs 1799983 gene polymorphisms could be a risk factor in the formation of migraine symptoms. In this respect, we recommend that to clarify the significance of this gene polymorphism and haplotype analysis, it is necessary to perform a large scale study and prospective cohort study with an increased number of subjects, to more accurately judge the association of the polymorphism with migraine.

Declaration of Interest. This research backed by the Trakya University Scientific Research Projects Unit, Edirne, Turkey. The authors report no conflicts of interest. The authors alone are responsible for the content and writing of this article.

\section{REFERENCES}

1. Borroni B, Rao R, Liberini P, Venturelli E, Cossandi M, Archetti S, et al. Endothelial nitric oxide synthase (Glu298Asp) polymorphism is an independent risk factor for migraine with aura. Headache. 2006; 46(10): 1575-1579.

2. Toriello M, Oterino A, Pascual J, Castillo J, Colás R, Alonso-Arranz A, et al. Lack of association of endothelial nitricoxide synthase polymorphisms and migraine. Headache. 2008; 48(7): 1115-1119.

3. Gruber HJ, Bernecker C, Lechner A, Weiss S, Wallner-Blazek m, Meinitzer A, et al. Increased nitric oxide stress is associated with migraine. Cephalalgia. 2010; 30(4): 486-492.

4. Gkaliagkousi E, Douma S, Zamboulis C, Ferro A. Nitric oxide dysfunction in vascular endothelium and platelets: Role in essential hypertension. J Hypertens. 2009; 27(12): 2310-2320.

5. Gonçalves FM, Martins-Oliveira A, Speciali JG, Luizon MR, Izidoro-Toledo TC, Silva PS, et $a l$. Endothelial nitric oxide synthase haplotypes associated with aura in patients with migraine. DNA Cell Biol. 2011; 30(6): 363-369.

6. Headache Classification Committee, International Headache Society. The International Classification of Headache Disorders, 2nd ed. Cephalalgia. 2004; 24(1): 1-160. 
7. Stovner, L, Hagen, K, Jensen, R, Katsarava Z, Lipton R, Scher A, et al. The global burden of headache: A documentation of headache prevalence and disability worldwide. Cephalalgia. 2007; 27(3): 193-210.

8. Gonçalves FM, Luizon MR, Speciali JG, Martins-Oliveira A, Dach F, Tanus-Santos JE, et al. Interaction among nitric oxide (NO)-related genes in migraine susceptibility. Mol Cell Biochem. 2012; 370(1-2): 183-189.

9. Olesen J. The role of nitric oxide (NO) in migraine, tension-type headache and cluster headache. Pharmacol Ther. 2008; 120(2): 157-171.

10. Olesen J. Nitric oxide-related drug targets in headache. Neurotherapeutics. 2010 7(2): 183-190.

11. Sarchielli P, Floridi A, Mancini ML, Rossi C, Coppola F, Baldi A, et al. NF-kB Activity and iNOS expression in monocytes from internal jugular blood of migraine without aura patients during attacks. Cephalalgia. 2006; 26(9): 10711079.

12. de O S Mansur T, Gonçalves FM, MartinsOliveira A, Speciali JG, Dach F, Lacchini R, et al. Inducible nitric oxide synthase haplotype associated with migraine and aura. Mol Cell Biochem. 2012; 364(1-2): 303-308.

13. Lea RA, Curtain RP, Shepherd AG, Brimage PJ, Griffiths LR. No evidence for involvement of the human inducible nitric oxide synthase (iNOS) gene in susceptibility to typical migraine. Am J Med Genet. 2001; 105(1): 110-113.

14. Jia S, Ni J, Chen S, Jiang Y, Dong W, Gao Y. Association of the pentanucleotide repeat polymorphism in NOS2 promoter region with susceptibility to migraine in a Chinese population. DNA Cell Biol. 2011; 30(2): 117-122.

15. Noseda R, Kainz V, Jakubowski M, Gooley JJ, Saper CB, Digre K, et al. A neural mechanism for exacerbation of headache by light. Nat Neurosci. 2010; 13(2): 239-245.

16. Milhaud D, Bogousslavsky J, van Melle G, Liot P. Ischemic stroke and active migraine. Neurology. 2001; 57(10): 1805-1811.

17. Olesen J. Nitric oxide-related drug targets in headache. Neurotherapeutics. 2010; 7(2): 183-190.

18. Napoli R, Guardasole V, Zarra E, Matarazzo M, D'Anna C, Saccà F, et al. Vascular smooth mus- cle cell dysfunction in patients with migraine. Neurology. 2009; 72(24): 2111-2114.

19. Myers DE. Potential neurogenic and vascular roles of nitric oxide in migraine headache and aura. Headache. 1999; 39(2): 118-124.

20. Tanus-Santos JE, Desai M, Flockhart DA. Effects of ethnicity on the distribution of clinically relevant endothelial nitric oxide variants. Pharmacogenetics. 2001; 1(8): 719-725.

21. Crawford DC, Nickerson DA. Definition and clinical importance of haplotypes. Annu Rev Med. 2005; 56(1): 303-320.

22. Metzger IF, Souza-Costa DC, Marroni AS, Nagassaki S, Desta Z, Flockhart DA, et al. Endothelial nitric oxide synthase gene haplotypes associated with circulating concentrations of nitric oxide products in healthy men. Pharmacogenet Genomics. 2005; 15(8): 565-570.

23. Wang J, Dudley D, Wang XL. Haplotype-specific effects on endothelial NO synthase promoter efficiency: Modifiable by cigarette smoking. Arterioscler Thromb Vasc Biol. 2002; 22(5): e1-e4.

24. Lauer T, Kleinbongard P, Kelm M. Indexes of NO bioavailability in human blood. News Physiol Sci. 2002; 17(6): 251-255.

25. Metzger IF, Sertorio JTC, Tanus-Santos JE. Relationship between systemic nitric oxide metabolites and cyclic GMP in healthy male volunteers. Acta Physiol. 2006; 188(2): 123-127.

26. Metzger IF, Sertório JT, Tanus-Santos JE. Modulation of nitric oxide formation by endothelial nitric oxide synthase gene haplotypes. Free Radic Biol Med. 2007; 43(6): 987-992.

27. Metzger IF, Ishizawa MH, Rios-Santos F, Carvalho WA, Tanus-Santos JE. Endothelial nitric oxide synthase gene haplotypes affect nitrite levels in black subjects. Phar-macogenomics J. 2011; 11(6): 393-399.

28. Metzger IF, Luizon MR, Lacchini R, Ishizawa MH, Tanus-Santos JE. Effects of endothelial nitric oxide synthase tagSNPs haplotypes on nitrite levels in Black subjects. Nitric Oxide. 2013; 28(1): 33-38.

29. Sipahi T, Guldiken B, Kabayel L, Palabıyık O, Oskan H, Kılıç TO, et al. Endothelial nitric oxide synthase and angiotensin converting enzyme gene polymorphisms in migraine patients. Arch Neuropsychiatr. 2013; 50(1): 274-278. 
30. Guldiken B, Sipahi T, Guldiken S, Ustundag S, Budak M, Turgut N, et al. Glu298Asp polymorphism of the endothelial nitric oxide synthase gene in Turkish patients with ischemic stroke. Mol Biol Rep. 2009; 36(6): 1539-1543.

31. Martelletti P, Lulli P, Morellini M, Mariani B, Pennesi G, Cappellacci S, et al. Chromosome 6pencoded HLA-DR2 determination discriminates migraine without aura from migraine with aura. Hum Immunol. 1999; 60(1): 69-74.
32. Rainero I, Grimaldi LM, Salani G, Valfrè W, Rivoiro C, Savi L, et al. Association between the tumor necrosis factor- $\alpha-308 \mathrm{G} /$ A gene polymorphism and migraine. Neurology. 2004; 62(1): 141-143.

33. Rubino E, Fenoglio P, Gallone S, Govone F, Vacca A, De Martino P, et al. Genetic variants in the NOTCH4 gene influence the clinical features of migraine. J Headache Pain. 2013; 14(1): 28-32. 